\title{
Enhancement of Plasmon Resonances in the Wave Scattering by Finite Grids of Circular Silver Wires
}

\author{
Denys M. Natarov ${ }^{1}$, Student Member, IEEE, Trevor M. Benson ${ }^{2}$, Senior Member, IEEE, \\ Ayhan Altintas $^{3}$, Senior Member, IEEE, Ronan Sauleau ${ }^{4}$, Senior Member, IEEE, \\ Alexander I. Nosich ${ }^{1}$, Fellow, IEEE \\ ${ }^{1}$ Institute of Radio-Physics and Electronics NASU, Kharkiv 61085, Ukraine \\ ${ }^{2} \mathrm{G}$. Green Institute for Electromagnetics Research, University of Nottingham, Nottingham NG7 2RD, UK \\ ${ }^{3}$ Bilkent University, Bilkent 06800, Ankara, Turkey \\ ${ }^{4}$ IETR, Universite de Rennes 1, Rennes Cedex 35042, France
}

\begin{abstract}
Using the field expansions in local coordinates and addition theorems for cylindrical functions, we consider the problem of the H-polarized plane wave scattered by a finite chain of circular wires. The absorption and scattering cross-sections are found numerically and plasmon and grating-type resonances are studied for the grids of silver nano-size wires.
\end{abstract}

Localized-surface-plasmon or electrostatic resonances are observed in the mid-infrared and optical bands [1]. It is known that small material objects can exhibit resonance behavior at certain frequencies for which the object permittivity is negative and the free-space wavelength is large in comparison to object dimensions. The physical mechanism behind these resonances is the presence of free electron gas in metal particles that may display collective oscillations that give the major contribution to the dielectric permittivity at optical frequencies. For subwavelength metallic objects, plasmon resonances result in powerful enhancement of scattered and absorbed light that is used in the design of optical antennas and biosensors for advanced applications. Plasmon resonances have unique physical property: in the leading terms, resonance frequencies depend on the object shape but do not depend on its geometric dimensions [2]. For example, they may shift considerably if the metal wires are collected in closely spaced ensembles, e.g. flat and curved grids, layers, and other finite-size configurations.

Ensembles of optically coupled nanoparticles and nanowires are important in today's research into the surface-enhanced Raman scattering of light where they are used as structured substrates. Finite structures made of periodic elements are even more interesting objects of research as they are strongly frequency-selective scatterers, i.e. their characteristics vary greatly with the frequency of the incident wave. As it is evident, here one of the major parameters is the relation between the period of the material structure and the wavelength however the size of elementary wire is also relevant. The frequency-selective behavior is usually based on the exploitation of resonances. As known, periodicity may lead to specific resonant phenomena. Among several kinds of resonances observed in periodic structures, important role is played by the so-called grating resonances [3]. Their frequencies are just below the Rayleigh frequencies [4,5] (period being a multiple of the wavelength) if the wires diameter is a fraction of the period.

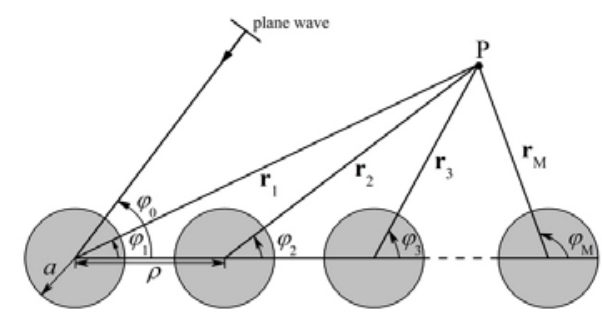

Fig. 1. Scattering problem and notations.

In the wave scattering by the infinite grids of wires, they lead to almost total reflection of the incident field by a sparse grid in a narrow frequency band. If the wires are non-magnetic, the Qfactors of resonances are much higher in the H-polarization case than in the E-polarization case. In [6] and [7], we have studied the formation of such a resonance in the scattering of the H-polarized plane waves by the large (hundreds of elements) albeit finite grids of sub-wavelength metallic and dielectric wires, respectively.

Continuing the research of [6,7], in this paper we study the influence of two kinds of the mentioned resonances on each other in the absorption and scattering characteristics, for two cases of the broadside and grazing light incidence on the structured nanosize scatterers.

Finite grid of equidistantly located parallel wires is shown in Fig. 1. The wires are assumed to be identical infinite circular cylinders, each having radius $a$ and complex relative dielectric permittivity $\varepsilon$. The distance between adjacent cylinders (i.e. period) is $\rho$ and their number is $M$. The global coordinates have the origin at the center of the first cylinder. For a 2-D problem, one has to find a scalar function $U$ that is either $E_{z}$ or $H_{z}$ scattered-field component. It must satisfy the Helmholtz equation, $\left(\Delta+k_{ \pm}^{2}\right) U^{ \pm}(\vec{r})=0$, where the wavenumbers are $k_{-}=\sqrt{\varepsilon} k_{0}$ inside of each cylinder and $k_{+}=k_{0}$ outside of all, the total-field tangential components continuity conditions, the 


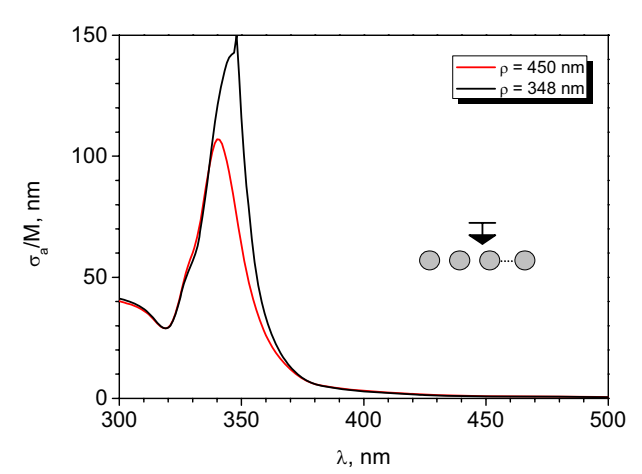

a)

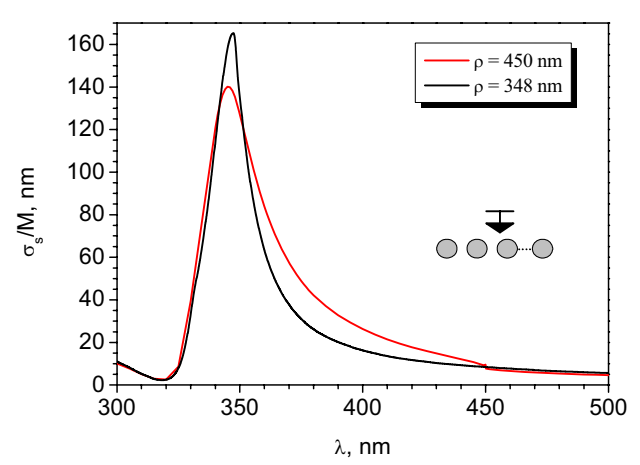

b)

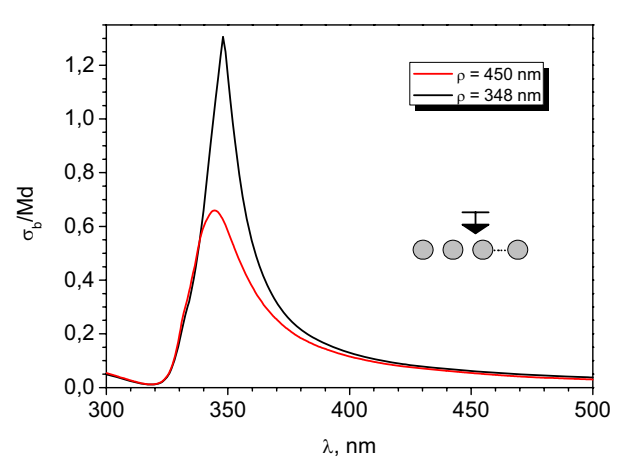

c)

Fig. 2. Normalized ACS (a), TCS (b) and BCS (c) as a function of wavelength for the $\mathrm{H}$-wave broadside incidence $\left(\varphi_{0}=90^{\circ}\right)$ on the grids of 800 silver nanowires with radius $a=25 \mathrm{~nm}$ and period $\rho=450$ and $348 \mathrm{~nm} ; d=(M-1) \rho+2 a$.

radiation condition at infinity, and the condition of the local power finiteness.

The solution can be obtained by expanding the field function in terms of the azimuth exponents in the local coordinates (Fig. 1), using addition theorems for cylindrical functions, and applying the boundary conditions on the surface of all $M$ cylinders. The unknown coefficients related to the $q$-th cylinder include the effect of all interactions between the cylinders $[6,7]$. They are finally reduced to an infinite matrix equation, $(I+U) X=U^{0}$, where $I$ is identity matrix, $X=\left(x_{m}^{(1)}, \ldots x_{m}^{(M)}\right)$ are unknown coefficients, and $U=\left\{\delta_{i j} U^{(i, j)}\right\}_{i, j=1, \ldots M}$ and $U^{0}=\left(U_{m}^{0(1)} . . U_{m}^{0(M)}\right), i, j=1, \ldots M, \quad m=0, \pm 1, \pm 2, \ldots$ are known functions of the wavelength and grid parameters. This is a $M \times M$ block-type Fredholm second kind equation, where each block is infinite. Thanks to this, the solution of corresponding counterpart equation with each block truncated to finite order $N$ converges to exact solution if $N \rightarrow \infty$. To test our code, we have compared it against numerical data of $[8,9]$; full agreement provides verification of our results. In $[4,5]$, we have investigated the grating and plasmon resonances for the light scattering by closely-spaced and sparse grids of dielectric and silver wires

On solving the mentioned matrix equation, one can find the scattered field at any point of space including the far-field scattering pattern, $\Phi(\varphi)$. The most important far-field characteristics are the absorption cross section (ACS), the total scattering cross section (TCS) and the backward cross section (BCS). They are computed as $\sigma_{a}=\operatorname{Im} \varepsilon k \int_{D}|\vec{E}(\vec{r})|^{2} d \vec{r}$, $\sigma_{s}=2 /(\pi k) \int_{0}^{2 \pi}|\Phi(\varphi)|^{2} d \varphi$, and $\sigma_{b}=4 / k \cdot\left|\Phi\left(\varphi_{0}\right)\right|^{2}$, where $D$ is the totality of the inner domains of all cylinders. Note that ACS can be also calculated from the optical theorem, $\sigma_{a}=-4 / k \cdot \operatorname{Re} \Phi\left(\varphi_{0}+\pi\right)-2 /(\pi k) \int_{0}^{2 \pi}|\Phi(\varphi)|^{2} d \varphi$.

In computations, we have considered the scattering of the $\mathrm{H}$ polarized plane waves by finite grids of parallel silver nanowires with radius about tens of nanometers, in the visible band. Note that for particles or wires as small as units of nanometers one can use the bulk experimental data published by Johnson and Christie [10] to characterize the complex dielectric permittivity of silver.

We know that plasmon resonance for a single silver wire with the radius $25 \mathrm{~nm}$ can be found at the $345 \mathrm{~nm}$ wavelength (see [1]). It is interesting what the peak values of the grid characteristics are if the plasmon and the grating resonance wavelengths coincide. Here, the main instrument of tuning them together is the period of the grid of metal nanowires.

In Fig. 2, presented are the absorption and scattering crosssections, normalized by the number of wires $M$, as functions of wavelength for the grids of 800 silver wires with radius $25 \mathrm{~nm}$ and two different periods: $450 \mathrm{~nm}$ and $348 \mathrm{~nm}$, under the broadside incidence. The grids with the both values of period are sparse, because $\rho \gg a$. If the period is detuned from the plasmon resonance, as with $\rho=450 \mathrm{~nm}$, then the cross section curves, after normalization by $M$, are very close to similar curves for a stand-alone circular silver nanowire. Computations performed with different grid periods tuned to plasmon resonant wavelength have shown that $\rho=348 \mathrm{~nm}$ gives the highest combined resonance in TCS. Fig. 2a shows that ACS for this value of $\rho$ increases to $150 \mathrm{~nm}$, i.e. by $40 \%$ with respect to $107 \mathrm{~nm}$ in the case of $\rho=450 \mathrm{~nm}$, and resonant wavelength is red-shifted from $340 \mathrm{~nm}$ to $348 \mathrm{~nm}$. Fig. $2 \mathrm{~b}$ shows that the total scattered power for $\rho=348 \mathrm{~nm}$ increases to $165.6 \mathrm{~nm}$, i.e. by $18 \%$ from $140.2 \mathrm{~nm}$ in the case of $\rho=450 \mathrm{~nm}$, and resonant wavelength is red-shifted from $345 \mathrm{~nm}$ to $348 \mathrm{~nm}$. Fig. 2c 


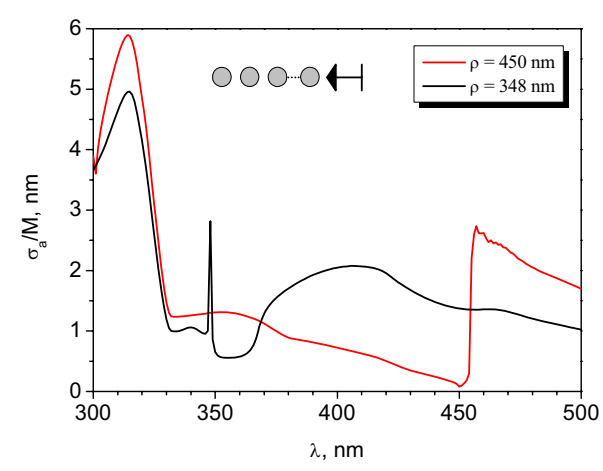

a)

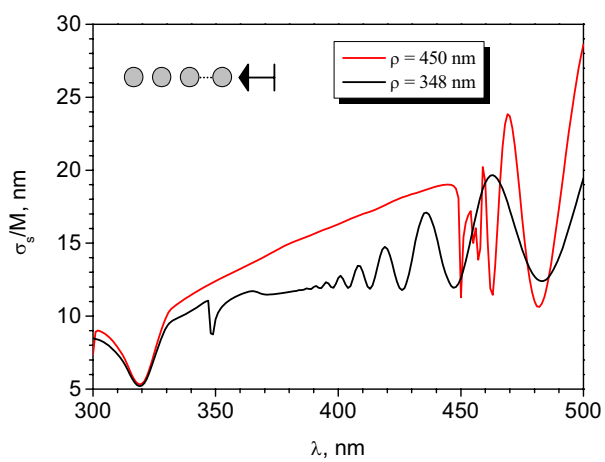

b)

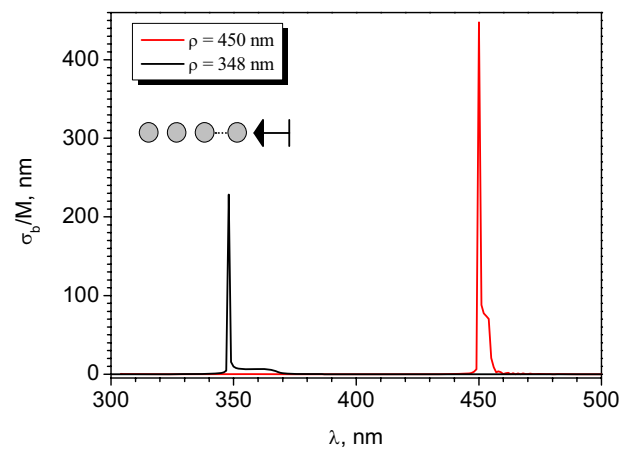

c)

Fig. 3. The same as in Fig. 2 for the H-wave grazing incidence $\left(\varphi_{0}=0^{0}\right)$.

shows that, if two resonances are tuned together (i.e. for $\rho=$ $348 \mathrm{~nm})$, BCS per one cylinder at the broadside illumination is approximately two times larger than for detuned resonances (for instance for $\rho=450 \mathrm{~nm}$ ), and resonant wavelength is redshifted from $345 \mathrm{~nm}$ to $348 \mathrm{~nm}$. Iin Fig. 2a one can see small narrow peak at the top of the plasmon resonance - this is the grating resonance.

In Fig. 3, one can see the absorption and scattering crosssections for the same grid parameters as in Fig. 2 however under the grazing incidence. In this case the plasmon resonance appears at $320 \mathrm{~nm}$, therefore the grating resonance linked to the period value is no more tuned to it for either grid. Unlike broadside incidence, here the both types of resonances produce not peaks but dips on the TCS plots. In Fig. 3c, one can see intensive and sharp grating resonances on the BCS curves for both period values - this is the first-order Bragg effect for discrete scatterers. Note that for all cross-sections the grating resonance Q-factor is higher for the grids with the 450 $\mathrm{nm}$ period. One can also see the oscillations in TCS if the wavelength becomes larger than the grid period.

Thus, in this paper we have presented accurate results for the absorption and scattering cross-sections for the H-polarized wave diffraction by the grids of 800 silver nanowires. We have investigated two types of resonances: plasmon and grating-type and computed two cases: of these resonances being well separated and being brought together. In the case of the broadside incidence, if plasmon and grating resonances are tuned together, resonance Q-factor increases. The highest increment of the resonant amplitude is observed for the BCS values under the grazing incidence of light.

This work has been partially supported by the European Science Foundation via the "Newfocus" network project.

\section{REFERENCES}

[1] J. P. Kottman, O. J. F. Martin, "Plasmon resonant coupling in metallic nanowires," Optics Express, vol. 8, no 12 , pp. 655-663, 2001.

[2] D. R. Fredkin, I. Mayergoyz, "Resonant behavior of dielectric objects (electrostatic resonances)," Physical Review Letters, vol. 91, no 25, pp. 39023905, 2003.

[3] R. Gomez-Medina, M. Laroche, J. J. Saenz, "Extraordinary optical reflection from subwavelength cylinder arrays," Optics Express, vol. 14, no. 9, pp. 3730-3737, 2006.

[4] R. W. Wood, "On a remarkable case of uneven distribution of light in a diffraction grating spectrum," Philosophical Magazine, vol. 4, pp. 396-403, 1902.

[5] Lord Rayleigh, "On the dynamical theory of gratings," Proc. Royal Soc. London, vol. A79, pp. 399-416, 1907

[6] D.M. Natarov, T.M. Benson, A. Altintas, R. Sauleau, A.I. Nosich, "Plasmon and grid resonances in the electromagnetic scattering by finite grids of silver nanowires", Proc. Int. Conf. Transparent Optical Networks (ICTON'10), Munich, 2010, Tu.P.8.

[7] D.M. Natarov, T.M. Benson, A. Altintas, R. Sauleau, A.I. Nosich, "Resonances in the electromagnetic scattering by very large finite-periodic grids of circular dielectric wires", Proc. Int. Kharkov Sym. on Physics and Engineering of Microwaves, MM and Sub-MM Waves (MSMW'10), Kharkiv, 2010, A-7.

[8] G. O. Olaofe, "Scattering by two cylinders," Radio Science, vol. 4, no. 11, pp. 1351-1 360, 1970.

[9] H. A. Ragheb, M. Hamid, "Scattering by N parallel conducting circular cylinders," Int. J. Electronics, vol. 59, no 4, pp. 407-421, 1985.

[10] P. B. Johnson, R. W. Christy, "Optical constants of the noble metals," Physical Review B, vol. 6, no 12, pp. $4370-4378,1972$. 